\title{
Receptor tyrosine kinases and schistosome reproduction: new targets for chemotherapy
}

\author{
Marion Morel ${ }^{1}$, Mathieu Vanderstraete ${ }^{1}$, Steffen Hahnel ${ }^{2}$, Christoph G. Grevelding ${ }^{2}$ and \\ Colette Dissous ${ }^{1}$ *
}

Center for Infection and Immunity of Lille, INSERM U1019, CNRS-UMR 8204, Institut Pasteur de Lille, University Lille Nord de France, Lille Cedex, France

2 Biomedical Centre for Research Seltersberg, Institute of Parasitology, Justus-Liebig-University Giessen, Giessen, Germany

Edited by:

Paul J. Brindley, The George

Washington University, USA

\section{Reviewed by:}

Paula Ribeiro, McGill University,

Canada

Emmitt Randolph Jolly, Case Western

Reserve University, USA

\section{${ }^{*}$ Correspondence:}

Colette Dissous, Center for Infection and Immunity of Lille, INSERM U1019, CNRS-UMR 8204, Institut

Pasteur de Lille, University Lille Nord de France, 1 Rue du Professeur

Calmette, 59019 Lille Cedex, France

e-mail:colette.dissous@pasteur-lille.fr
Schistosome parasites still represent a serious public health concern and a major economic problem in developing countries. Pathology of schistosomiasis is mainly due to massive egg production by these parasites and to inflammatory responses raised against the eggs which are trapped in host tissues. Tyrosine kinases (TKs) are key molecules that control cell differentiation and proliferation and they already represent important targets in cancer therapy. During recent years, it has been shown that receptor tyrosine kinases (RTK) signaling was active in reproductive organs and that it could regulate sexual maturation of schistosomes and egg production. This opens interesting perspectives for the control of transmission and pathogenesis of schistosomiasis based on new therapies targeting schistosome RTKs. This review relates the numerous data showing the major roles of kinase signaling in schistosome reproduction. It describes the conserved and particular features of schistosome RTKs, their implication in gametogenesis and reproduction processes and summarizes recent works indicating that RTKs and their signaling partners are interesting chemotherapeutical targets in new programs of control.

Keywords: schistosome, receptor tyrosine kinase, signaling, reproduction, chemotherapy

\section{SCHISTOSOMES, KINASE SIGNALING, AND REPRODUCTION}

Schistosomiasis, or bilharzia, is a parasitic disease with outstanding medical and economic importance, caused by trematodes of the Schistosoma genus (Chitsulo et al., 2004). Schistosoma mansoni, S. haematobium, and S. japonicum are the three major infective species in humans. Whereas most of the trematodes are hermaphrodites, schistosomes have separate sexes, and the sexual maturation of female worms is dependent on constant pairing with males (Popiel and Basch, 1984; LoVerde and Chen, 1991; Kunz, 2001). Pairing induces mitosis and maintains differentiation of stem cell-like precursor cells in the ovary and vitellarium (Erasmus, 1973; Den Hollander and Erasmus, 1985; Kunz, 2001; Galanti et al., 2012). These processes are a prerequisite for the production of eggs, formed by one oocyte and 30-40 vitelline cells combined within the ootype to form viable eggs. The control of this major parasitic disease currently relies on mass treatment with a single drug, Praziquantel. This drug is efficient against the three human schistosome species, but its widespread use raises fears about resistance and motivates the search for alternative therapies (Doenhoff et al., 2008; Melman et al., 2009). During recent years, much effort has been made to understand the development of female reproductive organs with the aim to find strategies to reduce transmission and morbidity of schistosomiasis by preventing egg production (LoVerde et al., 2009; Beckmann et al., 2010a). Indeed, eggs are responsible for parasite transmission but also for pathogenesis in humans since they accumulate in host tissues, particularly in liver, and elicit granulomatous inflammatory reactions leading to periportal fibrosis, portal hypertension, and hepatosplenomegaly (Hoffmann et al., 2002).
Evidence has been obtained that TGF- $\beta$ pathways play a major role in female reproductive development and egg embryogenesis. Essential components of TGF- $\beta$ pathways (TbRI and II, R-Smad, Co-Smad, FKBP12) have been identified in schistosomes, and their expression in vitelline cells was demonstrated (Davies et al., 1998; Beall et al., 2000; Osman et al., 2001, 2004, 2006; Knobloch et al., 2004). The TGF- $\beta$ pathway regulates the synthesis of SmGCP, a gynecophoral canal protein involved in promoting contact between males and females (Osman et al., 2006). The activin ligand of the receptor TbRII, SmInAct, is also crucial for successful embryogenesis in schistosome eggs (Freitas et al., 2007) and the TbRI serine/threonine kinase inhibitor (TRIKI) was shown to reduce vitelline cell mitotic activity and egg production in female worms (Knobloch et al., 2007). Tyrosine kinases (TKs) are also important for female gonad development and in this context, the Src-like cytosolic TK (CTK), SmTK3, seems to play a dominant role in proliferation of vitelline cells (Kapp et al., 2004). Treatment of parasites with the Src inhibitor Herbimycin A significantly blocked male-induced mitotic activity in paired females and interestingly, the combined treatment of paired schistosomes with TRIKI and Herbimycin A was more efficient to reduce mitosis and egg production, indicating possible cross-talk between Src and TGF- $\beta$ pathways (Knobloch et al., 2007). Recently, transcriptome analyses of inhibitor-treated schistosomes provided further evidence for a cooperation between Src-kinase and TGF- $\beta$ pathway in the control of mitosis and eggshell formation (Buro et al., 2013) and for an association of Abl-kinase activities with TGF $\beta$ signaling (Buro et al., 2014). Finally, the Syk kinase SmTK4 (Knobloch et al., 2002a,b) and the Src/Abl hybrid kinase SmTK6 
(Beckmann et al., 2011) presumably act together with SmTK3 in a multi-kinase complex to transduce signals potentially induced by the activation of membrane receptors, such as integrins $(\mathrm{Sm} \beta$ Int1) or receptor tyrosine kinases (RTKs) and which are important for gametogenesis in parasite gonads (Beckmann etal., 2011, 2012).

\section{SCHISTOSOME RECEPTOR TYROSINE KINASES}

Receptor tyrosine kinasess play essential roles in embryonic development and in various adult tissues and organs, in which they control fundamental processes, such as cell proliferation and differentiation, cell cycle and survival, migration, and metabolism (Ullrich and Schlessinger, 1990). RTKs form a superfamily of transmembrane proteins present in all metazoans, from sponges to humans (Suga et al., 2012) and they are composed of an extracellular ligand binding region formed by various subdomains, a single transmembrane domain, and an intracellular domain with intrinsic TK activity. Annotation of the S. mansoni genome (Berriman et al., 2009; Protasio et al., 2012) and analyses by combined computational approaches have shown that the tyrosine kinome of S. mansoni contains 15 RTKs including four members of the epidermal growth factor receptor (EGF-R) family, two of the IR (insulin receptor) family, two FGF-R (fibroblast growth factor receptor) members, one representative of Ephrin-R, ROR, and MuSK families, a homolog of CCK4, and one unknown receptor (Andrade et al., 2011; Avelar et al., 2011). Additionally, the schistosome genome encodes two Venus kinase receptors (VKRs), belonging to a novel family of RTKs originally discovered in $S$. mansoni (Vicogne et al., 2003; Dissous et al., 2014a,b).

Over the past few years, five RTKs of $S$. mansoni have been particularly well investigated. SER (Schistosoma EGF-R) was the first RTK described in S. mansoni (Shoemaker et al., 1992). It contains a conserved intracellular TK domain and an extracellular domain for binding of EGF ligands. When expressed in mammalian cells, SER can bind human EGF with the same affinity as human EGF-R (HER) and it activates the classical and conserved Ras/ERK signaling pathway. Human EGF was shown to induce SER autophosphorylation in S. mansoni adult worms and to increase protein and DNA synthesis as well as protein phosphorylation in schistosome larvae, indicating for the first time that host hormones were involved in regulating schistosome development (Vicogne et al., 2004). Such a potential dialog between host ligands and parasite receptors was further confirmed by the ability of human TGF- $\beta$ to activate TbRI/II schistosome receptors (Beall and Pearce, 2001) but also by evidence that human insulin can bind to schistosome membrane RTKs to regulate metabolic and glucose uptake activities in the parasite (Khayath et al., 2007; Ahier et al., 2008; You et al., 2010). Two members of the IR family were identified in S. mansoni (SmIR1 and SmIR2; Khayath et al., 2007), then in S. japonicum (SjIR1 and SjIR2; You et al., 2010). Schistosome IR1 and IR2 display differences in the structural motifs essential for signaling and in their expression sites. Schistosome IR1 is expressed in muscles, intestinal epithelial cells and at the basal membrane of the tegument (Khayath et al., 2007), and they are colocalized with SGTP1 and SGTP4, the schistosome glucose transporters involved in glucose uptake (Skelly et al., 1994). Schistosome IR2 is massively expressed in parenchymal cells of adult schistosomes (Khayath et al., 2007; You etal., 2010), and SjIR2 was also localized in vitelline cells (You et al., 2010). A single IR is present in most invertebrate species which regulates both metabolism and growth, while two receptors IR and IGF-1R exist in vertebrates, which are specialized in metabolic and glucose uptake regulation, and in growth control, respectively (Kim and Accili, 2002). It was suggested that similarly SmIR1 and SjIR1 could be specialized in sugar uptake and SmIR2 and SjIR2 preferentially involved in growth of schistosomes.

Besides these conventional and conserved RTKs, which are able to respond to host growth factors (EGF, insulin) and to activate conserved signaling pathways, schistosomes were shown to express unconventional RTKs, named VKRs for Venus kinase receptors (Vicogne et al., 2003; Ahier et al., 2009). VKRs are composed of an extracellular Venus flytrap module (VFT), linked through a single transmembrane fragment to an intracellular TK domain similar to that of IRs (Vicogne et al., 2003). VFTs are the extracellular domains of many G-protein coupled receptors of class $\mathrm{C}$, and they are composed of two lobes that close upon the binding of small ligands (amino-acids, ions) similarly to the leaves of the Venus flytrap carnivorous plant, Dionaea muscipula, when it catches its prey (Pin etal., 2003). VKRs are found in a large variety of invertebrates from cnidarians to echinoderms (Ahier et al., 2009; Vanderstraete et al., 2013a), and are highly expressed in larval stages and in gonads, suggesting a role of these proteins in embryonic and larval development as well as in reproduction (Ahier et al., 2009; Vanderstraete et al., 2014). Vkr genes are found as single copies in most species but in platyhelminths two different $v k r$ copies are present (Gouignard et al., 2012; Vanderstraete et al., 2013a, 2014). Up to now, two VKRs have been found in S. mansoni as well as in S. haematobium (Young et al., 2012). SmVKR1 and SmVKR2 of S. mansoni have been extensively studied. They were shown to be activated by L-arginine and calcium ions, respectively (Gouignard et al., 2012) and, as many other RTKs, VKRs dimerize to induce intracellular pathways involved in protein synthesis and cellular growth, like the MAPK and PI3K/Akt/S6K pathways (Dissous et al., 2014a; Vanderstraete et al., 2014).

Among the growth factor receptor panel, EGFR and IR/IGFR have been shown to play essential roles in mammals (Schneider and Wolf, 2008; Sirotkin, 2011) as well as in insects (Graf et al., 1997; Brown et al., 2008; Parrott et al., 2012) for the control of ovarian functions and reproductive processes. Additionally, the discovery that VKRs were abundantly transcribed in the gonads of many invertebrate species (Ahier et al., 2009), indicated that VKR signaling could also participate in reproductive activities. Further analyses have confirmed that all these RTKs were potentially involved in reproduction processes in schistosomes (Figure 1A).

\section{FUNCTIONS OF SCHISTOSOME RTKS IN REPRODUCTION}

Besides the importance of the receptor Ser/Thr (S/T) kinases SmTbRI/II and of TGF- $\beta$-dependent pathways in schistosome reproduction processes (LoVerde et al., 2007, 2009), TK signaling is also predominant for schistosome gonad development (Beckmann et al., 2010a,b), and a possible cooperation between STK and TK pathways has been proposed for the control of mitosis 


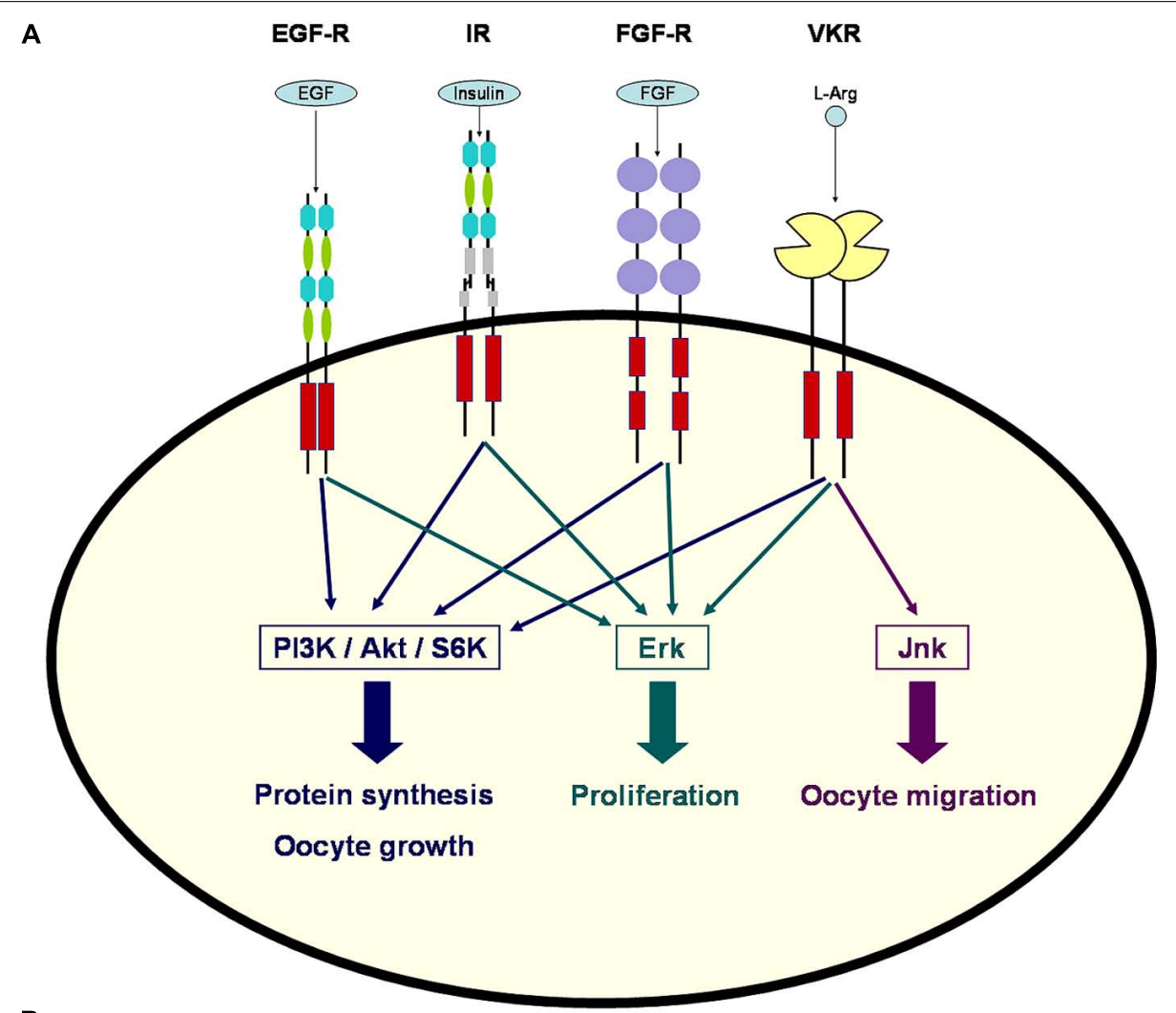

B

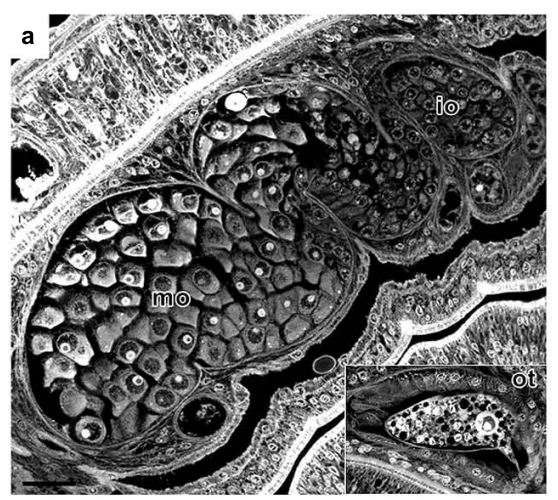

b W. Sar.

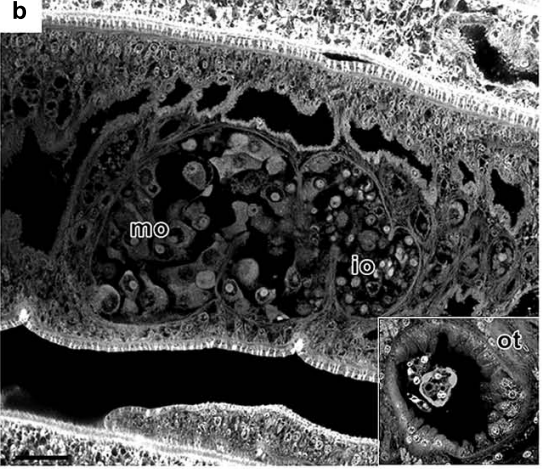

FIGURE 1 | Receptor tyrosine kinase (RTK) signaling in schistosome gonads. (A) Following activation by their respective ligands, schistosome RTKs (EGF-R, IR, FGF-R, and VKR) expressed in gonads can induce protein synthesis, cell growth and proliferation by activating the PI3K/AKt/S6K and Erk MAPK pathways. VKR activation was shown to also activate the Jnk MAPK pathway, potentially involved in oocyte migration. (B) Morphology of the reproductive organs of $S$. mansoni females is affected by ds $S m v k r$ RNA interference. Worms were treated with control irrelevant (A) or dsSmvkr (B)
dsRNAs (as described in Vanderstraete et al., 2014). Whole-mount preparations were stained with carmine red and examined by confocal laser scanning microscopy. In control worms (a), we observe immature oocytes (io) within the smaller, anterior part of the ovary and big and mature oocytes (mo) in the posterior part. The ootype (ot) contains a viable egg formed by one oocyte and vitelline cells. Treatment with dsSmvkr (b) induced a strong disorganization and size reduction of the ovary, as well as the abortion of egg formation. Scale bar: $20 \mu \mathrm{m}$. and eggshell formation in these parasites (Knobloch et al., 2006; Buro et al., 2013). Moreover, diverse CTKs of S. mansoni (SmTK3, SmTK4, and SmTK6) were shown to form complexes susceptible to participate in RTK signaling in gonads (Beckmann et al., 2011).

The EGF receptor SER is transcribed in the vitellarium and ovary of schistosome females together with its potential substrate SmEps8 (EGF-R kinase substrate 8) and SmTK3, the CTK that was shown to play a dominant role in proliferation of vitelline cells
(Kapp et al., 2004). Since SmEps8 interacts in yeast two-hybrid $(\mathrm{Y} 2 \mathrm{H})$ assays with SmTK3, this might indicate a possible role of SER and EGF pathways in reproductive activities (Beckmann et al., 2010a).

Insulin pathways, and especially the insulin-mediated PI3K pathway, play major roles in reproduction processes in many organisms (Graf et al., 1997; Brown et al., 2008). Moreover, the TOR (target of rapamycin)/S6K (p70 S6 kinase) pathway has 
been identified as an essential nutrient-sensing tool regulating egg development under the control of insulin in mosquitoes (Arsic and Guerin, 2008). Limited information has been obtained concerning the importance of IR receptors in the development of schistosome gonads, except that vaccination of mice with the purified insulin-binding domain of SjIR2 provoked a retardation of adult S. japonicum growth and a substantial decrease of egg maturation and laying in parasitized animals (You et al., 2012). While transcriptome analyses did not highlight an overexpression of IR transcripts neither in S. mansoni (Nawaratna et al., 2011) nor in S. japonicum (Gobert et al., 2009) gonads, they indicated an overexpression of VKR-like transcripts in ovary and vitelline cells of S. japonicum (Gobert et al., 2009). In S. mansoni, quantitative-PCR results confirmed that both Smvkr1 and Smvkr2 genes were more actively transcribed in female worms than in males (Gouignard et al., 2012). By in situ hybridization, Smvkr1 and Smvkr2 transcripts were detected in testes but they were more abundant in ovaries, in which the expression profile of each $v k r$ was noticeably different. Smvkr1 transcripts were present mainly in the posterior part of the ovary that contains mature oocytes (in prophase I of meiosis) whereas $5 m v k r 2$ transcripts were found in the anterior part of the organ containing immature oocytes. Q-PCR data obtained from isolated ovaries (Hahnel et al., 2013) indicated that both Smvkr1 and Smvkr2 transcripts were up-regulated strongly in the ovaries of sexually developed females as compared to the organs from virgin females issued from unisexual infections. Additionally, two RTKs of the FGFR family, SmFGFR-A and SmFGFR-B were found among the genes identified as up-regulated in female gonads after pairing (Hahnel et al., 2014, this issue). Analyses of isolated testes confirmed the presence of Smvkr1 and Smvkr2 transcripts in male reproductive organs and showed similarly their up-regulation in testes from males issued from bisexual infections (Vanderstraete et al., 2014). This indicated the particular importance of SmVKR receptors during the development and maturation of schistosome reproductive organs.

Recently, molecular partners of SmVKRs have been identified from $\mathrm{Y} 2 \mathrm{H}$ screening of an adult S. mansoni cDNA library with active intracellular domains of VKRs as baits. The analyses of the resulting partners indicated similarities between VKR and IR pathways, which is in agreement with the identities already observed within the intracellular domains of these RTKs. All the assumptions made from the nature of these partners about the specificity of phospho-pathways elicited by VKR, were supported by further studies of VKR activation and signaling in Xenopus oocytes. It was shown that ligand-activated VKRs induced, similarly to the endogenous insulin-activated Xenopus IR, the phosphorylation of Erk1/2, Akt, and p70S6K (Vanderstraete etal., 2014). This indicated that VKRs were able to stimulate protein synthesis and cellular growth, as IRs do. The JNK pathway was activated by SmVKR1 but not by SmVKR2, corroborating $\mathrm{Y} 2 \mathrm{H}$ screening results which showed a specific interaction of SmVKR1 with Rho1, Mek7, and PP2C (Vanderstraete et al., 2014). Since the JNK pathway has been shown to play a major role in oogenesis and meiosis resumption in Caenorhabditis elegans (Smith etal., 2002), in Drosophila melanogaster
A

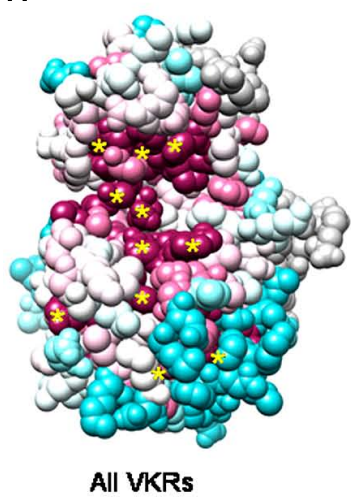

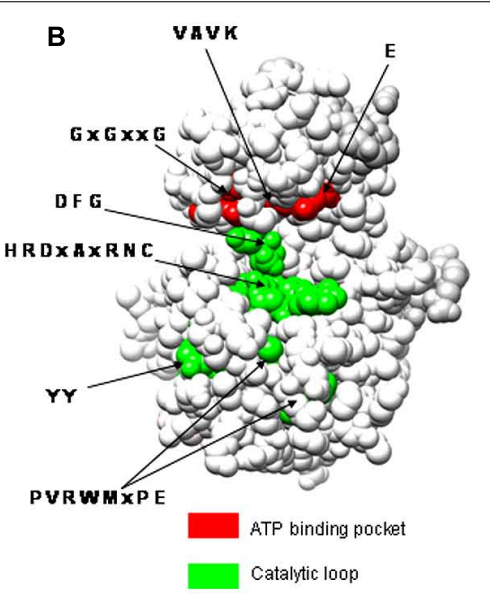

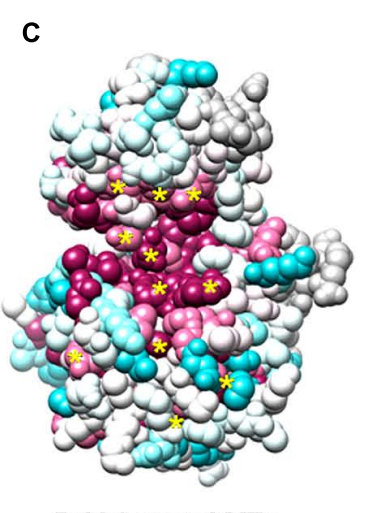

Schistosoma VKRs

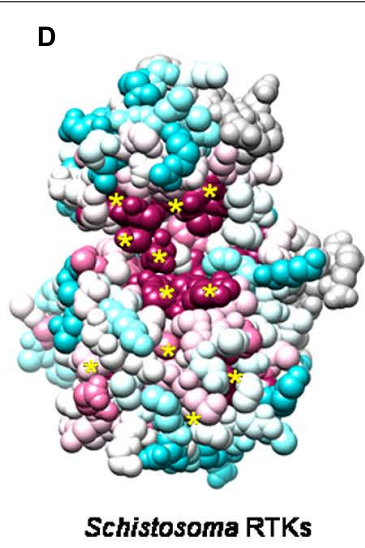

Schistosoma RTKs

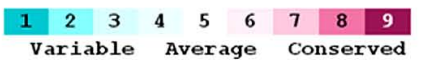

FIGURE 2 | Conservation of schistosome RTK tyrosine kinase catalytic sites. An evolutionary trace analysis of the conservation of residues in TK domains was performed using sequence alignment of (A) 40 VKRs already known in various invertebrate species Nanderstraete etal., 2013a), (C) four VKRs of Schistosoma (SmVKR1 AAL67949.1 and SmVKR2 ADD91576.1, and two VKRs of Schistosoma haematobium Sha_103537 and Sha_104501), (D) various Schistosoma RTKs (EGF, insulin and VKR receptors) including SmVKR1, SmVKR2, the two ShVKRs, SmIR1 (AAN39120), SmIR2 (AAV65745.2), SjIR1 (ACT20714.1), SjIR2 (ACT20715.1), and SER (AAA29866.1). Visualization of the conservation was performed on the human IR TK crystal structure (PDB accession number 1IRK; Ahier etal., 2009). The alignment generated with ClustalW was submitted to the ConSurf website server (http://consurf.tau.ac.il; Landau etal., 2005). Conservation scores of each residue were calculated by taking into account the phylogenetic relationships among the sequences and the similarity between the amino acids in the alignment. Conservation scores are according to a color scale from variable (blue) to conserved (purple) residues. In (B), are indicated the crucial residues of the ATP binding pocket (red) and of the catalytic loop (green) required for kinase activity. The evolutionary trace analyses revealed that these crucial residues (indicated by * in $\mathbf{A}, \mathbf{C}, \mathbf{D}$ ) are highly conserved among all VKRs $(\mathbf{A}, \mathbf{C})$ and among the panel of Schistosoma RTKs (EGF, insulin, and VKR receptors) from the three human species (mansoni, haematobium, and japonicum). 
(Sackton et al., 2007), and in mammals (Huang et al., 2011; Chuderland etal., 2012), it was postulated that it could be used as a pathway by SmVKR1 to influence oocyte maturation. Furthermore the implication of schistosome VKRs in oogenesis and spermatogenesis was demonstrated by RNA interference. SmVKR silencing led to an important disorganization of the anteroposterior structure of the ovary and the knock down of Smvkr1 resulted in the accumulation of big oocytes in the ovary and the absence of egg formation (Figure 1B). In male testes, silencing of both $S m v k r$ provoked a decrease of cell density within testicular lobes and paucity of sperm, confirming the potential importance of VKRs in reproduction processes (Vanderstraete et al., 2014).

\section{RTKS AS POTENTIAL TARGETS FOR THE CONTROL OF SCHISTOSOMIASIS}

Given the oncogenic role of aberrant signaling from RTKs in humans, these receptors have become attractive therapeutic targets. This led to the generation and the use in therapy of a large number of TK inhibitory compounds. Some of them were named as "tyrphostins" (TYRosine PHOSphorylation INnhibitors; Levitzki and Mishani, 2006), compounds that are able to inhibit multiple RTKs. We have seen that growth factor receptor and TK signaling molecules likely represent key molecules for the development and reproductive activity processes in schistosome worms. Consequently, these molecules are considered as potential targets for novel therapies against schistosomiasis today (Dissous et al., 2007; Dissous and Grevelding, 2011). IR inhibitors [tyrphostins AG1024, AG538, and HNMPA-(AM)3] can potentially affect survival of S. mansoni (Ahier et al., 2008) and S. japonicum (You et al., 2010) adult worms by blocking the uptake of glucose, an essential nutrient for schistosomes. Imatinib (Gleevec), used in the treatment of multiple cancers but targeting notably the chimeric oncogene BCR-Abl responsible for chronic myelogenous leukemia (Manley et al., 2002) has been described for its fatal impact on morphology, pairing stability, and survival of adult S. mansoni in vitro (Beckmann and Grevelding, 2010). Other TK inhibitors have also revealed a potential usefulness for the prevention of egg production by schistosomes. The Src kinase inhibitor, Herbimycin A, was demonstrated to block mitotic activity, expression of eggshell protein gene and egg production in S. mansoni female worms, preferentially inactivating the parasite Src-related SmTK3 and causing its degradation (Knobloch et al., 2006). The Syk inhibitor Piceatannol blocked the kinase activity of SmTK4 of S. mansoni and reduced egg production by female worms in vitro (Beckmann et al., 2010b). Furthermore, the angiokinase inhibitor BIBF1120 was shown to block the activities of the FGFR receptors SmFGFR-A and SmFGFR-B from $S$. mansoni leading to severe effects on the morphology of gonad tissues and the gastrodermis, on reproduction leading to reduced egg production, and finally on worm vitality (Hahnel et al., 2014, this issue).

More recently, tyrphostin AG1024 emerged as a potent drug molecule that caused dramatic effects both on the viability of larvae of $S$. mansoni and on the fertility of adult worms. The remarkable efficacy of this TK inhibitor (used at $\mu \mathrm{M}$ doses) on parasites was shown to be due to its dual action on the parasite IR and VKR kinases that contain similar catalytic domains (Vanderstraete et al., 2013b). Figure 2 illustrates the conservation of the catalytic pocket of RTKs characterized in the three human schistosome species. Using a sequence alignment of the TK domain of the various parasite RTKs and the crystal structure of the TK domain of the human insulin receptor, an evolutionary trace analysis revealed that in kinase domains the residues were highly conserved, and particularly those composing the catalytic loop and the ATP-binding site essential for kinase activity (Figures 2B-D). These residues were also identical among all the VKRs characterized in other invertebrate species (Figure 2A). The conservation of the motifs essential for TK activity in the various schistosome RTKs is very likely accounting for the efficacy of AG1024 to inhibit simultaneously schistosome IR, VKR but also EGFR kinase activities. This was confirmed by the high sensitivity of all these recombinant parasite kinases to AG1024 shown in kinase assays (Dissous et al., 2014b; Vanderstraete et al., 2014). In females of S. mansoni treated with AG1024, important size reduction and disorganization of the ovary were observed together with an inhibition of egg production. AG1024 also affected spermatogenesis in males, and all these data confirmed the potential of AG1024 to affect schistosome reproduction by targeting multiple RTKs. The implication of these RTKs in gametogenesis and reproduction processes are valuable reasons to consider them as interesting targets in new control programs, with the main advantage in the case of VKR that their counterparts are absent from the vertebrate host kinase panel. Structural divergences between catalytic domains of host and schistosome RTKs should now be exploited for the design of molecules able to target schistosome RTKs without affecting human host kinases. This should require for the parasite RTK kinase domains the obtention of X-ray crystal structures that would serve as the basis for designing new pharmaceuticals against schistosomiasis. Alternatively, the use of small molecules as antagonist ligands of the VFT domains of schistosome VKRs should be efficient to interfere specifically with VKR pathways and parasite reproduction.

\section{REFERENCES}

Ahier, A., Khayath, N., Vicogne, J., and Dissous, C. (2008). Insulin receptors and glucose uptake in the human parasite Schistosoma mansoni. Parasite 15, 573-579. doi: 10.1051/parasite/2008154573

Ahier, A., Rondard, P., Gouignard, N., Khayath, N., Huang, S., Trolet, J., et al. (2009). A new family of receptor tyrosine kinases with a Venus Flytrap binding domain in insects and other invertebrates activated by aminoacids. PLoS ONE 4:e5651. doi: 10.1371/journal.pone.0005651

Andrade, L., Nahum, L. A., Avelar, L. G. A., Silva, L. L., Zerlotini, A., Ruiz, J. C., et al. (2011). Eukaryotic protein kinases (ePKs) of the helminth parasite Schistosoma mansoni. BMC Genomics 12:215-234. doi: 10.1186/1471-2164-12-215

Arsic, D., and Guerin, P. M. (2008). Nutrient content of diet affects the signaling activity of the insulin/target of rapamycin/p70 S6 kinase pathway in the African malaria mosquito Anopheles gambiae. J. Insect Physiol. 54, 1226-1235. doi: 10.1016/j.jinsphys.2008.06.003

Avelar, L. G. A., Nahum, L. A., Andrade, L. F., and Oliveira, G. (2011). Functional diversity of the Schistosoma mansoni tyrosine kinases. J. Signal Transduct. 2011, 603290. doi: 10.1155/2011/603290

Beall, J., McGonigle, S., and Pearce, E. (2000). Functional conservation of Schistosoma mansoni Smads in TGF beta signaling. Mol. Biochem. Parasitol. 111, 131-142. doi: 10.1016/S0166-6851(00)00307-8

Beall, M. J., and Pearce, E. J. (2001). Human transforming growth factor-beta activates a receptor serine/threonine kinase from the intravascular parasite Schistosoma mansoni. J. Biol. Chem. 276, 31613-31619. doi: 10.1074/jbc.M104685200 
Beckmann, S., and Grevelding, C. G. (2010). Imatinib has a fatal impact on morphology, pairing stability and survival of adult Schistosoma mansoni in vitro. Int. J. Parasitol. 40, 521-526. doi: 10.1016/j.ijpara.2010.01.007

Beckmann, S., Hahnel, S., Cailliau, K., Vanderstraete, M., Browaeys, E., Dissous, C., et al. (2011). Characterization of the Src/Abl hybrid kinase SmTK6 of Schis tosoma mansoni. J. Biol. Chem. 286, 42325-42336. doi: 10.1074/jbc.M110. 210336

Beckmann, S., Quack, T., Burmeister, C., Buro, C., Long, T., Dissous, C., et al. (2010a). Schistosoma mansoni: signal transduction processes during the development of the reproductive organs. Parasitology 137, 497-520. doi: $10.1017 /$ S0031182010000053

Beckmann, S., Buro, C., Dissous, C., Hirzmann, J., and Grevelding, C. G. (2010b). The Syk kinase SmTK4 of Schistosoma mansoni is involved in the regulation of spermatogenesis and oogenesis. PLoS Pathog. 6:e1000769. doi: 10.1371/journal.ppat.1000769

Beckmann, S., Quack, T., Dissous, C., Cailliau, K., Lang, G., and Grevelding, C. G. (2012). Discovery of platyhelminth-specific $\alpha / \beta$-integrin families and evidence for their role in reproduction in Schistosoma mansoni. PLoS ONE 7:e52519. doi: 10.1371/journal.pone.0052519

Berriman, M., Haas, B. J., LoVerde, P. T., Wilson, R. A., Dillon, G. P., Cerqueira, G. C., et al. (2009). The genome of the blood fluke Schistosoma mansoni. Nature 460, 352-358. doi: 10.1038/nature08160

Brown, M. R., Clark, K. D., Gulia, M., Zhao, Z., Garczynski, S. F., Crim, J. W. et al. (2008). An insulin-like peptide regulates egg maturation and metabolism in the mosquito Aedes aegypti. Proc. Natl. Acad. Sci. U.S.A. 105, 5716-5721. doi: 10.1073/pnas.0800478105

Buro, C., Beckmann, S., Oliveira, K. C., Dissous, C., Cailliau, K., Marhöfer, R. J., et al. (2014). Imatinib treatment causes substantial transcriptional changes in adult Schistosoma mansoni in vitro exhibiting pleiotropic effects. PLoS Negl. Trop. Dis. 8:e2923. doi: 10.1371/journal.pntd.0002923

Buro, C., Oliveira, K. C., Lu, Z., Leutner, S., Beckmann, S., Dissous, C., et al. (2013) Transcriptome analyses of inhibitor-treated schistosome females provide evidence for cooperating Src-kinase and TGF $\beta$ receptor pathways controlling mitosis and eggshell formation. PLoS Pathog. 9:e1003448. doi: 10.1371/journal.ppat. 1003448

Chitsulo, L., LoVerde, P., and Engels, D. (2004). Schistosomiasis. Nat. Rev. Microbiol. 2, 12-13. doi: 10.1038/nrmicro801

Chuderland, D., Dvashi, Z., Kaplan-Kraicer, R., Ben-Meir, D., Shalgi, R., and Lavi, S. (2012). De novo synthesis of protein phosphatase 1A, magnesium dependent, alpha isoform (PPM1A) during oocyte maturation. Cell. Mol. Biol. Lett. 17, 433-445. doi: 10.2478/s11658-012-0022-7

Davies, S. J., Shoemaker, C. B., and Pearce, E. J. (1998). A divergent member of the transforming growth factor beta receptor family from Schistosoma manson is expressed on the parasite surface membrane. J. Biol. Chem. 273, 11234-11240. doi: $10.1074 /$ jbc.273.18.11234

Den Hollander, J. E., and Erasmus, D. A. (1985). Schistosoma mansoni: male stimulation and DNA synthesis by the female. Parasitology 91, 449-457. doi: 10.1017/S0031182000062697

Dissous, C., Ahier, A., and Khayath, N. (2007). Protein tyrosine kinases as new potential targets against human schistosomiasis. Bioessays 29, 1281-1288. doi: 10.1002/bies.20662

Dissous, C., and Grevelding, C. G. (2011). Piggy-backing the concept of cancer drugs for schistosomiasis treatment: a tangible perspective? Trends Parasitol. 27, 59-66. doi: 10.1016/j.pt.2010.09.001

Dissous, C., Morel, M., and Vanderstraete, M. (2014a). Venus kinase receptors: prospects in signaling and biological functions of these invertebrate receptors. Front. Endocrinol. (Lausanne) 5:72. doi: 10.3389/fendo.2014.00072

Dissous, C., Vanderstraete, M., Beckmann, S., Gouignard, N., Leutner, S., Buro, C., et al. (2014b). "Receptor tyrosine kinase signaling and drug targeting in schistosomes," in Protein Phosphorylation in Parasites: Novel Targets for Antiparasitic Intervention, eds C. Doerig, G. Späth, and M. Wiese (Weinheim: Wiley-VCH Verlag GmbH \& Co.KgaA), 337-356.

Doenhoff, M. J., Cioli, D., and Utzinger, J. (2008). Praziquantel: mechanisms of action, resistance and new derivatives for schistosomiasis. Curr. Opin. Infect. Dis 21, 659-667. doi: 10.1097/QCO.0b013e328318978f

Erasmus, D. A. (1973). A comparative study of the reproductive system of mature, immature and "unisexual" female Schistosoma mansoni. Parasitology 67, 165-183. doi: $10.1017 /$ S0031182000046394
Freitas, T. C., Jung, E., and Pearce, E. J. (2007). TGF-beta signaling controls embryo development in the parasitic flatworm Schistosoma mansoni. PLoS Pathog. 3:e52. doi: 10.1371/journal.ppat.0030052

Galanti, S. E., Huang, S. C., and Pearce, E. J. (2012). Cell death and reproductive regression in female Schistosoma mansoni. PLoS Negl. Trop. Dis. 6:e1509. doi: 10.1371/journal.pntd.0001509

Gobert, G. N., McManus, D. P., Nawaratna, S., Moertel, L., Mulvenna, J., and Jones, M. K. (2009). Tissue specific profiling of females of Schistosoma japonicum by integrated laser microdissection microscopy and microarray analysis. PLoS Negl. Trop. Dis. 3:e469. doi: 10.1371/journal.pntd.0000469

Gouignard, N., Vanderstraete, M., Cailliau, K., Lescuyer, A., Browaeys, E., and Dissous, C. (2012). Schistosoma mansoni: structural and biochemical characterization of two distinct venus kinase receptors. Exp. Parasitol. 132, 32-39. doi: 10.1016/j.exppara.2011.05.007

Graf, R., Neuenschwander, S., Brown, M. R., and Ackermann, U. (1997). Insulinmediated secretion of ecdysteroids from mosquito ovaries and molecular cloning of the insulin receptor homologue from ovaries of bloodfed Aedes aegypti. Insect Mol. Biol. 6, 151-163. doi: 10.1111/j.1365-2583.1997.tb00083.x

Hahnel, S., Lu, Z., Wilson, R. A., Grevelding, C. G., and Quack, T. (2013). Wholeorgan isolation approach as a basis for tissue-specific analyses in Schistosoma mansoni. PLoS Negl. Trop. Dis. 7:e2336. doi: 10.1371/journal.pntd.0002336

Hahnel, S., Quack, T., Parker-Manuel, S. J., Lu, Z., Vanderstraete, M., Morel, M., et al. (2014). Gonad RNA-specific qRT-PCR analyses identify genes with potential functions in schistosome reproduction such as SmFzl and SmFGFRs. Front. Genet. 5:170. doi: 10.3389/fgene.2014.00170

Hoffmann, K. F., Wynn, T. A., and Dunne, D. A. (2002). Cytokine-mediated host responses during schistosome infections; walking the fine line between immunological control and immunopathology. Adv. Parasitol. 52, 265-307. doi: 10.1016/S0065-308X(02)52014-5

Huang, X., Tong, J. S., Wang, Z. B., Yang, C. R., Qi, S. T., Guo, L., et al. (2011). JNK2 participates in spindle assembly during mouse oocyte meiotic maturation. Microsc. Microanal. 7, 197-205. doi: 10.1017/S1431927610094456

Kapp, K., Knobloch, J., Schussler, P., Sroka, S., Lammers, R., Kunz, W., et al. (2004). The Schistosoma mansoni Src kinase TK3 is expressed in the gonads and likely involved in cytoskeletal organization. Mol. Biochem. Parasitol. 138, 171-182. doi: 10.1016/j.molbiopara.2004.07.010

Khayath, N., Vicogne, J., Ahier, A., Ben Younes, A., Konrad, C., Trolet, J., et al. (2007). Diversification of the insulin receptor family in the helminth parasite Schistosoma mansoni. FEBS J. 274, 659-676. doi: 10.1111/j.1742-4658.2006.05610.x

Kim, J. J., and Accili, D. (2002). Signaling through IGF-1 and insulin receptors: where is the specificity? Growth Horm. IGF Res. 12, 84-90. doi: 10.1054/ghir.2002.0265

Knobloch, J., Beckmann, S., Burmeister, C., Quack, T., and Grevelding, C. G. (2007). Tyrosine kinase and cooperative TGFbeta signaling in the reproductive organs of Schistosoma mansoni. Exp. Parasitol. 117, 318-336. doi: 10.1016/j.exppara.2007.04.006

Knobloch, J., Kunz, W., and Grevelding, C. G. (2002a). Quantification of DNA synthesis in multicellular organisms by a combined DAPI and BrdU technique. Dev. Growth Differ. 44, 559-563. doi: 10.1046/j.1440-169X.2002.00667.x

Knobloch, J., Winnen, R., Quack, M., Kunz, W., and Grevelding, C. G. (2002b). A novel Syk-family tyrosine kinase from Schistosoma mansoni which is preferentially transcribed in reproductive organs. Gene 294, 87-97. doi: 10.1016/S0378-1119(02)00760-6

Knobloch, J., Kunz, W., and Grevelding, C. G. (2006). Herbimycin A suppresses mitotic activity and egg production of female Schistosoma mansoni. Int. J. Parasitol. 36, 1261-72. doi: 10.1016/j.ijpara.2006.06.004

Knobloch, J., Rossi, A., Osman, A., LoVerde, P. T., Klinkert, M. Q., and Grevelding, C. G. (2004). Cytological and biochemical evidence for a gonad-preferential interplay of SmFKBP12 and SmTbetaR-I in Schistosoma mansoni. Mol. Biochem. Parasitol. 138, 227-236. doi: 10.1016/j.molbiopara.2004.09.006

Kunz, W. (2001). Schistosome male-female interaction: induction of germ-cell differentiation. Trends Parasitol. 17, 227-231. doi: 10.1016/S1471-4922(01)01893-1

Landau, M., Mayrose, I., Rosenberg, Y., Glaser, F., Martz, E., Pupko, T., et al. (2005). ConSurf 2005: the projection of evolutionary conservation scores of residues on protein structures. Nucleic Acids Res. 33, W299-W302. doi: 10.1093/nar/ gki370

Levitzki, A., and Mishani, E. (2006). Tyrphostins and other tyrosine kinase inhibitors. Annu. Rev. Biochem. 75, 93-109. doi: 10.1146/annurev.biochem.75.10 3004.142657 
LoVerde, P. T., Andrade, L. F., and Oliveira, G. (2009). Signal transduction regulates schistosome reproductive biology. Curr. Opin. Microbiol. 12, 422-428. doi: 10.1016/j.mib.2009.06.005

LoVerde, P. T., and Chen, L. (1991). Schistosome female reproductive development. Parasitol. Today 7, 303-308. doi: 10.1016/0169-4758(91)90263-N

LoVerde, P. T., Osman, A., and Hinck, A. P. (2007). Schistosoma mansoni: TGF-b signaling pathways. Exp. Parasitol. 117, 304-317. doi: 10.1016/j.exppara.2007.06.002

Manley, P. W., Cowan-Jacob, S. W., Buchdunger, E., Fabbro, D., Fendrich, G., Furet, P., et al. (2002). Imatinib: a selective tyrosine kinase inhibitor. Eur. J. Cancer 38(Suppl 5), S19-S27. doi: 10.1016/S0959-8049(02)80599-8

Melman, S. D., Steinauer, M. L., Cunningham, C., Kubatko, L. S., Mwangi, I. N., Wynn, N. B., et al. (2009). Reduced susceptibility to praziquantel among naturally occurring Kenyan isolates of Schistosoma mansoni. PLoS Negl. Trop. Dis. 3:e504. doi: 10.1371/journal.pntd.0000504

Nawaratna, S. S., McManus, D. P., Moertel, L., Gobert, G. N., and Jones, M. K. (2011). Gene atlasing of digestive and reproductive tissues in Schistosoma mansoni. PLoS Negl. Trop. Dis. 5:e1043. doi: 10.1371/journal.pntd.0001043

Osman, A., Niles, E. G., and LoVerde, P. T. (2001). Identification and characterization of a Smad2 homologue from Schistosoma mansoni, a transforming growth factor-beta signal transducer. J. Biol. Chem. 276, 10072-10082. doi: 10.1074/jbc.M005933200

Osman, A., Niles, E. G., and LoVerde, P. T. (2004). Expression of functional Schistosoma mansoni Smad4: role in Erk-mediated transforming growth factor beta (TGF-beta) down-regulation. J. Biol. Chem. 279, 6474-6486. doi: 10.1074/jbc.M310949200

Osman, A., Niles, E. G., Verjovski-Almeida, S., and LoVerde, P. T. (2006). Schistosoma mansoni TGF-beta receptor II: role in host ligand-induced regulation of a schistosome target gene. PLoS Pathog. 2:e54. doi: 10.1371/journal.ppat.0020054

Parrott, B. B., Hudson, A., Brady, R., and Schulz, C. (2012). Control of germline stem cell division frequency - a novel, developmentally regulated role for epidermal growth factor signaling. PLoS ONE 7:e36460. doi: 10.1371/journal.pone. 0036460

Pin, J. P., Galvez, T., and Prézeau, L. (2003). Evolution, structure, and activation mechanism of family 3/C G-protein-coupled receptors. Pharmacol. Ther. 98 325-354. doi: 10.1016/S0163-7258(03)00038-X

Popiel, I., and Basch, P. F. (1984). Reproductive development of female Schistosoma mansoni (Digenea: Schistosomatidae) following bisexual pairing of worms and worm segments. J. Exp. Zool. 232, 141-150. doi: 10.1002/jez.1402320117

Protasio, A. V., Tsai, I. J., Babbage, A., Nichol, S., Hunt, M., Aslett, M. A., et al. (2012). A systematically improved high quality genome and transcriptome of the human blood fluke Schistosoma mansoni. PLoS Negl. Trop. Dis. 6:e1455. doi 10.1371/journal.pntd.0001455

Sackton, K. L., Buehner, N. A., and Wolfner, M. F. (2007). Modulation of MAPK activities during egg activation in Drosophila. Fly (Austin) 1, 222-227.

Schneider, M. R., and Wolf, E. (2008). The epidermal growth factor receptor and its ligands in female reproduction: insights from rodent models. Cytokine Growth Factor Rev. 19, 173-181. doi: 10.1016/j.cytogfr.2008.01.003

Shoemaker, C. B., Ramachandran, H., Landa, A., dos Reis, M. G., and Stein, L. D. (1992). Alternative splicing of the Schistosoma mansoni gene encoding a homologue of epidermal growth factor receptor. Mol. Biochem. Parasitol. 53, 17-32. doi: 10.1016/0166-6851(92)90003-3

Sirotkin, A. V. (2011). Growth factors controlling ovarian functions. J. Cell. Physiol. 226, 2222-2225. doi: 10.1002/jcp.22588

Skelly, P. J., Kim, J. W., Cunningham, J., and Shoemaker, C. B. (1994). Cloning, characterization and functional expression of cDNAs encoding glucose transporter proteins from the human parasite Schistosoma mansoni. J. Biol. Chem. 269, $4247-4253$
Smith, P., Leung-Chiu, W. M., Montgomery, R., Orsborn, A., Kuznicki, K. GressmanCoberly, E., et al. (2002). The GLH proteins, Caenorhabditis elegans $\mathrm{P}$ granule components, associate with CSN-5 and KGB-1, proteins necessary for fertility, and with ZYX-1, a predicted cytoskeletal protein. Dev. Biol. 251, 333-347. doi: 10.1006/dbio.2002.0832

Suga, H., Dacre, M., de Mendoza, A., Shalchian-Tabrizi, K., Manning, G., and RuizTrillo, I. (2012). Genomic survey of premetazoans shows deep conservation of cytoplasmic tyrosine kinases and multiple radiations of receptor tyrosine kinases. Sci. Signal. 5:ra35. doi: 10.1126/scisignal.2002733

Ullrich, A., and Schlessinger, J. (1990). Signal transduction by receptors with tyrosine kinase activity. Cell 61, 203-212. doi: 10.1016/0092-8674(90)90801-K

Vanderstraete, M., Gouignard, N., Ahier, A., Morel, M., Vicogne, J., and Dissous, C. (2013a). The venus kinase receptor (VKR) family: structure and evolution. BMC Genomics 14:361. doi: 10.1186/1471-2164-14-36

Vanderstraete, M., Gouignard, N., Cailliau, K., Morel, M., Lancelot, J., Bodart, J. F., et al. (2013b). Dual targeting of insulin and venus kinase Receptors of Schistosoma mansoni for novel anti-schistosome therapy. PLoS Negl. Trop. Dis. 7:e2226. doi: 10.1371/journal.pntd.0002226

Vanderstraete, M., Gouignard, N., Cailliau, K., Morel, M., Hahnel, S., Leutner, S., et al. (2014). Venus kinase receptors control reproduction in the platyhelminth parasite Schistosoma mansoni. PloS Pathog. 10:e1004138. doi: 10.1371/journal.ppat. 1004138

Vicogne, J., Cailliau, K., Tulasne, D., Browaeys, E., Yan, Y., Fafeur, V., et al. (2004). Conservation of epidermal growth factor receptor function in the human parasitic helminth Schistosoma mansoni. J. Biol. Chem. 279, 37407-37414. doi: 10.1074/jbc.M313738200

Vicogne, J., Pin, J. P., Lardans, V., Capron, M., Noël, C., and Dissous, C. (2003). An unusual receptor tyrosine kinase of Schistosoma mansoni contains a Venus Flytrap module. Mol. Biochem. Parasitol. 126, 51-62. doi: 10.1016/S0166-6851(02) 00249-9

You, H., Gobert, G. N., Duke, M. G., Zhang, W., Li, Y., Jones, M. K., et al. (2012). The insulin receptor is a transmission blocking veterinary vaccine target for zoonotic Schistosoma japonicum. Int. J. Parasitol. 42, 801-807. doi: 10.1016/j.ijpara.2012.06.002

You, H., Zhang, W., Jones, M. K., Gobert, G., Mulvenna, J., Rees, G., et al. (2010). Cloning and characterization of Schistosoma japonicum insulin receptors. PLoS ONE 5:e9868. doi: 10.1371/journal.pone.0009868

Young, N. D., Jex, A. R., Li, B., Liu, S., Yang, L., Xiong, Z., et al. (2012) Wholegenome sequence of Schistosoma haematobium. Nat. Genet. 44, 221-225. doi: 10.1038/ng.1065

Conflict of Interest Statement: The authors declare that the research was conducted in the absence of any commercial or financial relationships that could be construed as a potential conflict of interest.

Received: 26 May 2014; accepted: 03 July 2014; published online: 18 July 2014. Citation: Morel M, Vanderstraete M, Hahnel S, Grevelding CG and Dissous C (2014) Receptor tyrosine kinases and schistosome reproduction: new targets for chemotherapy. Front. Genet. 5:238. doi: 10.3389/fgene.2014.00238

This article was submitted to Evolutionary and Genomic Microbiology, a section of the journal Frontiers in Genetics.

Copyright (C) 2014 Morel, Vanderstraete, Hahnel, Grevelding and Dissous. This is an open-access article distributed under the terms of the Creative Commons Attribution License (CC BY). The use, distribution or reproduction in other forums is permitted, provided the original author(s) or licensor are credited and that the original publication in this journal is cited, in accordance with accepted academic practice. No use, distribution or reproduction is permitted which does not comply with these terms. 